\title{
APPROXIMATE CONTROLLABILITY OF LINEAR PARABOLIC EQUATIONS IN PERFORATED DOMAINS
}

\author{
PATrizia DONATO ${ }^{1}$ AND AÏSSAM NABIL ${ }^{2}$
}

\begin{abstract}
In this paper we consider an approximate controllability problem for linear parabolic equations with rapidly oscillating coefficients in a periodically perforated domain. The holes are $\varepsilon$-periodic and of size $\varepsilon$. We show that, as $\varepsilon \rightarrow 0$, the approximate control and the corresponding solution converge respectively to the approximate control and to the solution of the homogenized problem. In the limit problem, the approximation of the final state is alterated by a constant which depends on the proportion of material in the perforated domain and is equal to 1 when there are no holes. We also prove that the solution of the approximate controllability problem in the perforated domain behaves, as $\varepsilon \rightarrow 0$, as that of the problem posed in the perforated domain having as rigth-hand side the (fixed) control of the limit problem.
\end{abstract}

Mathematics Subject Classification. 35K05, 49A50, 93C20.

Received March 2, 1999. Revised January 7, July 10 and September 8, 2000.

\section{INTRODUCTION}

In this paper we study the asymptotic behaviour, as $\varepsilon \rightarrow 0$, of an approximate controllability problem for linear parabolic equations with rapidly oscillating coefficients in a perforated domain. The holes in the perforated domain are $\varepsilon$-periodic and of size $\varepsilon$. The oscillations of the coefficients are also of order $\varepsilon$.

By definition, one has approximate controllability if the set of reachable final states is dense in $L^{2}$. Following Lions [12], we construct, for a fixed $\varepsilon$, an approximate internal control as the (unique) solution of a related transposed problem (see Sect. 4). The final data of this problem is the (unique) minimum point of a suitable functional $J_{\varepsilon}$. We study then the asymptotic behavior of the system as $\varepsilon$ goes to zero.

In Section 3 we state the main result of this paper (Th. 3.4). It shows that the approximate control and the corresponding solution converge respectively to the control and to the solution of the homogenized problem. This means that the control problem in the highly heterogeneous system may be replaced by the homogenized one, which might be relevant from a computational point of view.

At the limit, the approximation of the desired final state is altered by a constant $\frac{1}{\sqrt{\theta}} \geq 1$, where $\theta$ represents the proportion of the material in the perforated domain. When there are no holes this constant is equal to 1, which gives the result proved by Zuazua [14].

We also show (Cor. 3.7) that one can replace, in the problem posed in the perforated domain, the approximate control by the approximate control of the homogenized problem.

Keywords and phrases: Linear parabolic equation, approximate controlability, homogenization.

${ }^{1}$ Université de Rouen, UFR des Sciences, UPRES-A 6085 du CNRS, Site Colbert, 76821 Mont-Saint-Aignan, France; e-mail: donato@univ-rouen.fr and Laboratoire d'Analyse Numérique, Université Paris VI, Case Postale 187, 4 place Jussieu, 75252 Paris Cedex 05, France; e-mail: donato@ann.jussieu.fr

2 ENSA Agadir, Université Ibn Zohr, BP. 33/S, Agadir, Morocco. 
In Sections 4 and 5 we construct, for a fixed $\varepsilon$, the approximate control of the system in the perforated domain and we give some a priori estimates. To do that, we adapt to our case some methods introduced by Fabre et al. in [10] and [11], where they prove the approximate controllability of the semilinear heat equation with Dirichlet conditions. We also follow some ideas from Zuazua [14], where he studies the asymptotic behaviour of the heat equation with oscillating coefficients in a fixed domain.

The proof of the main result is given in Section 7 . For passing to the limit as $\varepsilon \rightarrow 0$, we use some results on the homogenization and correctors for linear parabolic equations in perforated domains proved by the authors in [9]. We recall them in Section 2. The main point of the proof is to identify the limit of the controls. We do that in Section 6 by using De Giorgi's $\Gamma$-convergence techniques (see Props. 6.4 and 6.5). We prove that the minimum point of $J_{\varepsilon}$ converge, as $\varepsilon$ tends to zero, to the (unique) minimum point of a suitable functional $J$. Moreover, this functional is that associated to the homogenized problem in the construction of the approximate control. One of the main difficulties coming from the presence of the holes is that one cannot simply use the lower semicontinuity of the $L^{2}$-norm in order to identify the limit control. Lemma 6.6 in Section 6 allows to overcome this difficulty.

The results of this paper were announced in [8].

\section{Preliminaries}

Let $\Omega$ be a bounded connected open set of $\mathbb{R}^{n}, n \geq 2$, with boundary $\partial \Omega$ of class $\mathcal{C}^{2}$. Let $Y=] 0, l_{1}[\times \cdots \times] 0, l_{n}$ [ be the reference cell and $S \subset \subset Y$ an open subset (the reference hole) with boundary $\partial S$ of class $\mathcal{C}^{2}$. We denote by $\varepsilon$ a positive parameter taking its values in a positive sequence which tends to zero. Introduce the set of holes in $\mathbb{R}^{n}$ defined by

$$
\tau(\varepsilon \bar{S})=\left\{\varepsilon(k(l)+\bar{S}), k \in \mathbb{Z}^{n}, k(l)=\left(k_{1} l_{1}, \cdots, k_{n} l_{n}\right)\right\}
$$

Assume that the sequence $(\varepsilon)$ and the open set $\Omega$ are such that

$$
\partial \Omega \cup \tau(\varepsilon \bar{S})=\emptyset
$$

This means that there exists a subset $\mathcal{K}_{\varepsilon}$ of $\mathbb{Z}^{n}$ such that

$$
\Omega \cap \tau(\varepsilon \bar{S})=\cup_{k \in \mathcal{K}_{\varepsilon}}(\varepsilon(k(l)+\bar{S})) .
$$

Set

Then, the perforated domain $\Omega_{\varepsilon}$ is defined by

$$
S_{\varepsilon}=\cup_{k \in \mathcal{K}_{\varepsilon}}(\varepsilon(k(l)+\bar{S})) .
$$

$$
\Omega_{\varepsilon}=\Omega \backslash S_{\varepsilon}
$$

From (2.1), we have then

In the following we use the notations:

$$
\partial \Omega \cap \partial S_{\varepsilon}=\emptyset, \quad \partial \Omega_{\varepsilon}=\partial \Omega \cup \partial S_{\varepsilon} .
$$

- $Y^{*}=Y \backslash \bar{S}$;

- $|\omega|=$ the Lesbegue measure of a measurable set $\omega$ of $\mathbb{R}^{n}$;

- $\theta=\left|Y^{*}\right| /|Y|$

- $\chi_{\omega}=$ the characteristic function of the set $\omega, \chi_{\omega}(x)=\left\{\begin{array}{cc}1 & \text { if } x \in \omega \\ 0 & \text { elsewhere }\end{array}\right.$;

- $\widetilde{v}=$ the extension by zero on $\Omega$ of any function $v$ defined on $\Omega_{\varepsilon}$;

- $\nu=\left(\nu_{i}\right)_{i=1, \ldots, n}$ the unit external normal vector with respect to $Y \backslash \bar{S}$ or $\Omega_{\varepsilon}$.

In the following we denote by $c$ different constants independent of $\varepsilon$.

Recall that (for a proof see for instance [3], Chap. 2), as $\varepsilon \rightarrow 0$,

$$
\chi_{\Omega_{\varepsilon}} \rightarrow \theta=\left|Y^{*}\right| /|Y| \quad L^{\infty}(\Omega) \text { weak } * .
$$


This is due to fact that, by assumption (2.1), one has

$$
\chi_{\Omega_{\varepsilon}}(x)=\left(\chi_{Y^{*}}\right)^{\#}\left(\frac{x}{\varepsilon}\right)
$$

where $\left(\chi_{Y^{*}}\right)^{\#}$ is defined by

$$
\left(\chi_{Y^{*}}\right)^{\#}\left(y+k l_{i} e_{i}\right)=\chi_{Y^{*}}(y) \quad \text { a.e. on } Y, \quad \forall k \in \mathbb{Z}, \quad \forall i \in\{1, \ldots, n\},
$$

and $\left\{e_{1}, \ldots, e_{n}\right\}$ is the canonical basis of $\mathbb{R}^{n}$.

Let $A(y)=\left(a_{i j}(y)\right)_{1 \leq i, j \leq n}$ be a $n \times n$ matrix-valued function defined on $\mathbb{R}^{n}$ such that

$$
\left\{\begin{array}{l}
A \in\left(C^{1}(\bar{Y})\right)^{n^{2}}, a_{i j}=a_{j i} \quad \forall 1 \leq i, j \leq n, \\
A \quad \text { is } Y \text {-periodic, } \\
\text { there exists } \alpha>0 \text { such that for any } \lambda=\left(\lambda_{1}, \ldots, \lambda_{n}\right) \in \mathbb{R}^{n}, \\
\sum_{i, j=1}^{n} a_{i j}(y) \lambda_{i} \lambda_{j} \geq \alpha\|\lambda\|^{2} \quad \text { a.e. on } Y,
\end{array}\right.
$$

and denote for any $\varepsilon$,

Let $V_{\varepsilon}$ be the Hilbert space

$$
A^{\varepsilon}(x)=A\left(\frac{x}{\varepsilon}\right) \quad \text { a.e. on } \Omega
$$

equipped with the $H^{1}\left(\Omega_{\varepsilon}\right)$-norm.

$$
V_{\varepsilon}=\left\{v \in H^{1}\left(\Omega_{\varepsilon}\right): v_{\left.\right|_{\partial \Omega}}=0\right\}
$$

Let us consider the following problem:

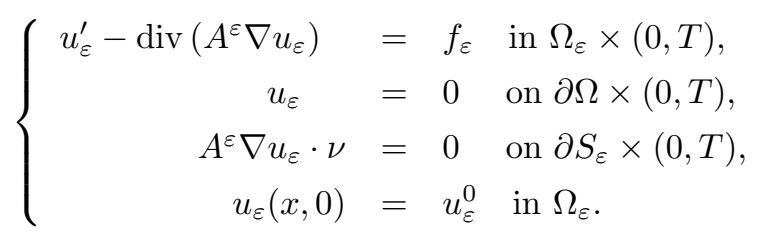

It is well known (see [5], Chap. XVIII, Sect. 3) that if $f_{\varepsilon} \in L^{2}\left(\Omega_{\varepsilon} \times(0, T)\right)$ and $u_{\varepsilon}^{0} \in L^{2}\left(\Omega_{\varepsilon}\right)$, problem (2.4) has a unique solution $u_{\varepsilon}$ such that

$$
u_{\varepsilon} \in L^{2}\left(0, T ; V_{\varepsilon}\right) \cap C^{0}\left([0, T] ; L^{2}\left(\Omega_{\varepsilon}\right)\right)
$$

In the following, we will make use of some homogenization results for linear parabolic problems in $\Omega_{\varepsilon}$, proved in [9]. We recall them here for the reader's convenience.

We make the following assumptions, as $\varepsilon \rightarrow 0$ :

$$
\left\{\begin{array}{rr}
\text { i) } & \widetilde{u_{\varepsilon}^{0}} \rightarrow u^{0} \quad \text { weakly in } L^{2}(\Omega), \\
\text { ii) } & \widetilde{f}_{\varepsilon} \rightarrow f \quad \text { weakly in } L^{2}(\Omega \times(0, T)) .
\end{array}\right.
$$

Let us introduce the homogenized matrix $A^{0}$, which is the same as in the elliptic case studied in [4]. For any $\lambda \in \mathbb{R}^{n}$, let $\widehat{\chi}_{\lambda}$ be the solution of the following problem:

$$
\left\{\begin{array}{c}
-\operatorname{div}\left(A \nabla\left(y \cdot \lambda-\widehat{\chi}_{\lambda}\right)\right)=0 \text { in } Y^{*} \\
\left(A \nabla\left(y \cdot \lambda-\widehat{\chi}_{\lambda}\right)\right) \cdot \nu=0 \text { on } \partial S \\
\widehat{\chi}_{\lambda} \text { Y-periodic } \\
\int_{Y^{*}} \widehat{\chi}_{\lambda}(y) d y=0
\end{array}\right.
$$


where $A$ is the matrix given by (2.3). Set

$$
\widehat{w}^{\lambda}(y)=-\widehat{\chi}_{\lambda}(y)+\lambda \cdot y \quad \text { a.e. on } Y^{*} .
$$

Then the $n \times n$ homogenized matrix $A^{0}=\left\{a_{i j}^{0}\right\}_{1 \leq i, j \leq n}$ is defined by

$$
A^{0} \lambda=\frac{1}{|Y|} \int_{Y^{*}} A \nabla \widehat{w}^{\lambda} d y, \quad \text { for any } \lambda \in \mathbb{R}^{n} .
$$

We also introduce the $(n \times n) Y$-periodic corrector matrix, $C(y)=\left\{C_{i j}(y)\right\}_{1 \leq i, j \leq n}$ where $C_{i j}(y)$ is defined by

with

$$
C_{i j}(y)=\delta_{i j}(y)-\frac{\partial \widehat{\chi}^{j}}{\partial y_{i}}(y)=\frac{\partial \widehat{w}^{j}}{\partial y_{i}}(y) \quad \text { a.e. } \quad \text { on } Y^{*},
$$

$$
\widehat{w}^{j}=x_{j}-\widehat{\chi}^{j},
$$

where $\widehat{\chi}^{j}$ is the solution of (2.7) for $\lambda=e_{j}$ and $\delta_{i j}$ is the Kronecker symbol.

We define

$$
C^{\varepsilon}(x)=C\left(\frac{x}{\varepsilon}\right), \text { a.e. on } \Omega_{\varepsilon},
$$

which, by construction, is $\varepsilon Y$-periodic.

We have the following result (see [9]):

Theorem 2.1. Under hypothesis (2.3) and (2.6), let $u_{\varepsilon}$ be the solution of problem (2.4). Then, there exists an extension operator $P^{\varepsilon} \in \mathcal{L}\left(L^{2}\left(0, T ; V_{\varepsilon}\right) ; L^{2}\left(0, T ; H_{0}^{1}(\Omega)\right)\right)$, such that, as $\varepsilon \rightarrow 0$, the following convergences hold:

$$
\begin{aligned}
& \text { i) } \quad P^{\varepsilon} u_{\varepsilon} \rightarrow u \quad \text { weakly in } L^{2}\left(0, T ; H_{0}^{1}(\Omega)\right) \text {, } \\
& \text { ii) } \widetilde{A^{\varepsilon} \nabla u_{\varepsilon}} \rightarrow A^{0} \nabla u \quad \text { weakly in }\left[L^{2}(\Omega \times(0, T))\right]^{n} \text {, }
\end{aligned}
$$

where $u$ is the solution of the homogenized equation

$$
\left\{\begin{array}{clll}
\theta u^{\prime}-\operatorname{div}\left(A^{0} \nabla u\right) & = & f & \text { in } \Omega \times(0, T), \\
u & = & 0 & \text { on } \partial \Omega \times(0, T), \\
u(x, 0) & = & \frac{u^{0}}{\theta} \quad \text { in } \Omega,
\end{array}\right.
$$

with $A^{0}$ given by (2.9).

Moreover, as $\varepsilon \rightarrow 0$, we have the following convergences:

$$
\left\{\begin{array}{ccl}
\text { i) } & \widetilde{u_{\varepsilon}} \rightarrow \theta u & \text { weakly in } L^{\infty}\left(0, T ; L^{2}(\Omega)\right), \\
\text { ii) } & \widetilde{u_{\varepsilon}} \longrightarrow \theta u & \text { strongly in } C^{0}\left([0, T] ; H^{-1}(\Omega)\right) .
\end{array}\right.
$$

If further we suppose that

$$
\begin{gathered}
\lim _{\varepsilon \rightarrow 0}\left\|f_{\varepsilon}-\frac{1}{\theta} f\right\|_{L^{2}\left(\Omega_{\varepsilon} \times(0, T)\right)}=0, \\
\lim _{\varepsilon \rightarrow 0}\left\|u_{\varepsilon}^{0}-\frac{1}{\theta} u^{0}\right\|_{L^{2}\left(\Omega_{\varepsilon}\right)}=0,
\end{gathered}
$$

we have

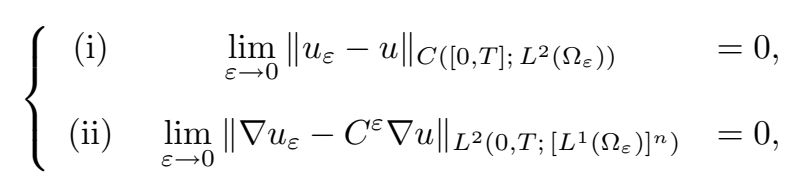

where $C^{\varepsilon}$ is the corrector matrix defined by (2.10). 
Remark 2.2. i) For the construction of the extension operator $P^{\varepsilon}$ we refer to [1] and [2] (see also [4]).

ii) Assumption (2.15) is equivalent to the following two conditions (see for instance [8], Lem. 5.4), as $\varepsilon \rightarrow 0$ :

$$
\begin{aligned}
& \widetilde{u_{\varepsilon}^{0}} \rightarrow u^{0} \quad \text { weakly in } L^{2}(\Omega), \\
& \left\|u_{\varepsilon}^{0}\right\|_{L^{2}\left(\Omega_{\varepsilon}\right)}^{2} \longrightarrow \frac{1}{\theta}\left\|u^{0}\right\|_{L^{2}(\Omega)}^{2} .
\end{aligned}
$$

Observe also that, if (2.15) holds, then for any sequence $\left\{\varphi_{\varepsilon}\right\}_{\varepsilon} \subset L^{2}\left(\Omega_{\varepsilon}\right)$ such that

$$
\widetilde{\varphi_{\varepsilon}} \rightarrow \varphi \text { weakly in } L^{2}(\Omega) \text {, }
$$

as $\varepsilon \rightarrow 0$, one has

$$
\lim _{\varepsilon \rightarrow 0} \int_{\Omega_{\varepsilon}} u_{\varepsilon}^{0} \varphi_{\varepsilon} d x=\int_{\Omega} \frac{1}{\theta} u^{0} \varphi d x
$$

\section{MAin RESUlts}

In this section we state the main results of this paper. They will be proved in the next sections. Let $\omega$ be an open nonempty subset of $\Omega$ and set

$$
\omega_{\varepsilon}=\omega \cap \Omega_{\varepsilon} .
$$

We can always assume that $\left|\omega \cap \Omega_{\varepsilon}\right| \neq 0$, for any $\varepsilon>0$. We consider the following approximate controllability system:

$$
\left\{\begin{aligned}
u_{\varepsilon}^{\prime}-\operatorname{div}\left(A^{\varepsilon} \nabla u_{\varepsilon}\right) & =\chi_{\omega_{\varepsilon}} \phi_{\varepsilon} & & \text { in } \Omega_{\varepsilon} \times(0, T), \\
u_{\varepsilon} & =0 & & \text { on } \partial \Omega \times(0, T), \\
A^{\varepsilon} \nabla u_{\varepsilon} \cdot \nu & =0 & & \text { on } \partial S_{\varepsilon} \times(0, T), \\
u_{\varepsilon}(x, 0) & =u_{\varepsilon}^{0} & & \text { in } \Omega_{\varepsilon},
\end{aligned}\right.
$$

where $u_{\varepsilon}^{0} \in L^{2}\left(\Omega_{\varepsilon}\right)$ and $\phi_{\varepsilon} \in L^{2}\left(\Omega_{\varepsilon} \times(0, T)\right)$.

Definition 3.1. We say that we have approximate controllability for system $(3.1)$ in $L^{2}\left(\Omega_{\varepsilon}\right)$ at time $T>0$ if the following holds:

For every $u_{\varepsilon}^{0} \in L^{2}\left(\Omega_{\varepsilon}\right)$, the set $E(T)$ of the reachable states at time $T>0$ defined by

$$
E(T)=\left\{u_{\varepsilon}(x, T), u_{\varepsilon} \text { is solution of (3.1) with } \phi_{\varepsilon} \in L^{2}\left(\omega_{\varepsilon} \times(0, T)\right)\right\}
$$

is dense in $L^{2}\left(\Omega_{\varepsilon}\right)$.

In other words:

Given $T>0$, for any $u_{\varepsilon}^{0} \in L^{2}\left(\Omega_{\varepsilon}\right), \alpha>0$ and for any $w_{\varepsilon}^{1} \in L^{2}\left(\Omega_{\varepsilon}\right)$, there exists $\phi_{\varepsilon} \in L^{2}\left(\omega_{\varepsilon} \times(0, T)\right)$ such that the corresponding solution of (3.1) satisfies

$$
\left\|u_{\varepsilon}(x, T)-w_{\varepsilon}^{1}\right\|_{L^{2}\left(\Omega_{\varepsilon}\right)} \leq \alpha .
$$

Remark 3.2. Let $\phi_{\varepsilon}^{0}$ be given in $L^{2}\left(\Omega_{\varepsilon}\right)$. From the unique continuation principle (see [13]), applied to the solution of the transposed heterogeneous problem

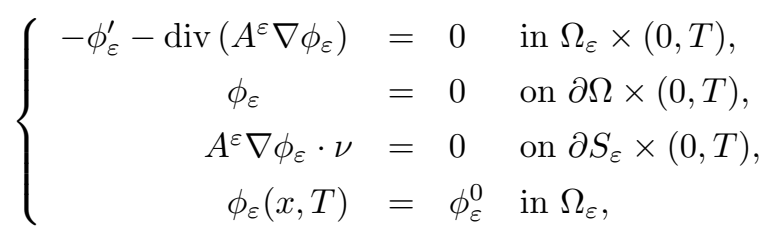


one has that if

$$
\phi_{\varepsilon}=0 \quad \text { in } \omega_{\varepsilon} \times(0, T)
$$

then

$$
\phi_{\varepsilon}=0 \quad \text { in } \Omega_{\varepsilon} \times(0, T) .
$$

Hence, for $\varepsilon$ fixed, the approximate controllability of system (3.1) follows by using classical duality arguments. But this method is not constructive.

A general method for constructing an approximate control was introduced by Lions [12]. The idea is to built the control as the solution of a transposed problem associated to some initial condition. This initial condition is obtained as the minimum point of a suitable functional associated to the problem. Here, we construct a functional $J_{\varepsilon}$ as in Zuazua [14] (see also Fabre et al. [10] and [11]). To do that, for $u_{\varepsilon}^{0} \in L^{2}\left(\Omega_{\varepsilon}\right)$ let us introduce the solution $v_{\varepsilon}$ of the problem

$$
\left\{\begin{array}{clll}
v_{\varepsilon}^{\prime}-\operatorname{div}\left(A^{\varepsilon} \nabla v_{\varepsilon}\right) & = & 0 & \text { in } \Omega_{\varepsilon} \times(0, T), \\
v_{\varepsilon} & = & 0 & \text { on } \partial \Omega \times(0, T), \\
A^{\varepsilon} \nabla v_{\varepsilon} \cdot \nu & = & 0 & \text { on } \partial S_{\varepsilon} \times(0, T), \\
v_{\varepsilon}(x, 0) & = & u_{\varepsilon}^{0} & \text { in } \Omega_{\varepsilon} .
\end{array}\right.
$$

Let now $w_{\varepsilon}^{1} \in L^{2}\left(\Omega_{\varepsilon}\right)$ be given. For any $\phi_{\varepsilon}^{0} \in L^{2}\left(\Omega_{\varepsilon}\right)$, we set

$$
J_{\varepsilon}\left(\phi_{\varepsilon}^{0}\right)=\frac{1}{2} \int_{0}^{T} \int_{\omega_{\varepsilon}}\left|\phi_{\varepsilon}\right|^{2} d x d t+\alpha\left\|\phi_{\varepsilon}^{0}\right\|_{L^{2}\left(\Omega_{\varepsilon}\right)}-\int_{\Omega_{\varepsilon}}\left(w_{\varepsilon}^{1}-v_{\varepsilon}(T)\right) \phi_{\varepsilon}^{0} d x
$$

where $\phi_{\varepsilon}$ is the solution of (3.3) (remark that $w_{\varepsilon}^{1}-v_{\varepsilon}(T)$ is still in $L^{2}\left(\Omega_{\varepsilon}\right)$, since $w_{\varepsilon}^{1}$ is in $L^{2}\left(\Omega_{\varepsilon}\right)$ and $v_{\varepsilon}$ is in $C\left([0, T] ; L^{2}\left(\Omega_{\varepsilon}\right)\right)$.

The following theorem gives the approximate controllability for $\varepsilon$ fixed.

Theorem 3.3. Given $T>0$, under hypothesis (2.3), let $\alpha>0, u_{\varepsilon}^{0} \in L^{2}\left(\Omega_{\varepsilon}\right)$ and $w_{\varepsilon}^{1} \in L^{2}\left(\Omega_{\varepsilon}\right)$ be fixed. Then $J_{\varepsilon}$ has a unique minimum point $\Phi_{\varepsilon}^{0}$. Moreover, if $\Phi_{\varepsilon}$ is the solution of (3.3) for $\phi_{\varepsilon}^{0}=\Phi_{\varepsilon}^{0}$, then the solution $u_{\varepsilon}$ of the following system:

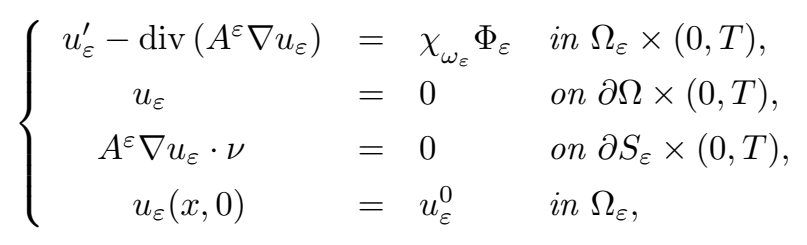

satisfies the estimate:

$$
\left\|u_{\varepsilon}(T)-w_{\varepsilon}^{1}\right\|_{L^{2}\left(\Omega_{\varepsilon}\right)} \leq \alpha
$$

This result will be proved in Section 4. In Section 5 (Cor. 5.3 and Rem. 5.4) we give some a priori estimates on the controls. They will allow, together with the homogenization results quoted in Section 2, to describe the asymptotic behaviour of problem (3.6).

To do that, let us introduce, for $u^{0} \in L^{2}(\Omega)$, the solution $v$ of the problem

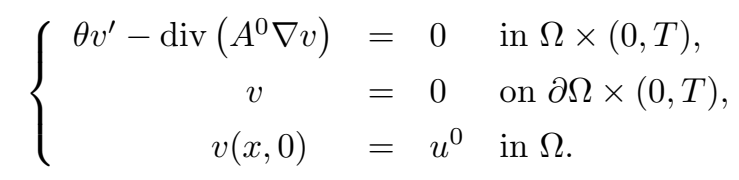

For a given $w^{1} \in L^{2}(\Omega)$, we define the functional $J$ on $L^{2}(\Omega)$ by

$$
J\left(\phi^{0}\right)=\frac{1}{2} \theta \int_{0}^{T} \int_{\omega}|\phi|^{2} d x d t+\alpha \sqrt{\theta}\left\|\phi^{0}\right\|_{L^{2}(\Omega)}-\theta \int_{\Omega}\left(w^{1}-v(T)\right) \phi^{0} d x,
$$


for any $\phi^{0} \in L^{2}(\Omega)$, where $\phi$ is the solution of the following homogeneous transposed problem:

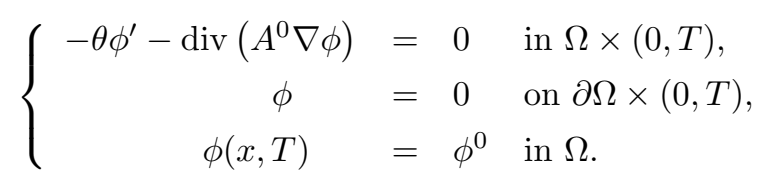

The main result of this paper is the following:

Theorem 3.4. Let $T>0$ be given. Under hypothesis (2.3), let also $\alpha>0, u^{0} \in L^{2}(\Omega)$ and $w^{1} \in$ $L^{2}(\Omega)$ be given. Suppose that $\left\{w_{\varepsilon}^{1}\right\}_{\varepsilon} \subset L^{2}\left(\Omega_{\varepsilon}\right)$ and $\left\{u_{\varepsilon}^{0}\right\}_{\varepsilon} \subset L^{2}\left(\Omega_{\varepsilon}\right)$ satisfy the following assumptions:

$$
\text { i) } \quad \begin{aligned}
& \lim _{\varepsilon \rightarrow 0}\left\|w_{\varepsilon}^{1}-w^{1}\right\|_{L^{2}\left(\Omega_{\varepsilon}\right)}=0, \\
& \text { ii) } \quad \lim _{\varepsilon \rightarrow 0}\left\|u_{\varepsilon}^{0}-u^{0}\right\|_{L^{2}\left(\Omega_{\varepsilon}\right)}=0 .
\end{aligned}
$$

Let $u_{\varepsilon}(x, t)$ be the solution of the controllability problem:

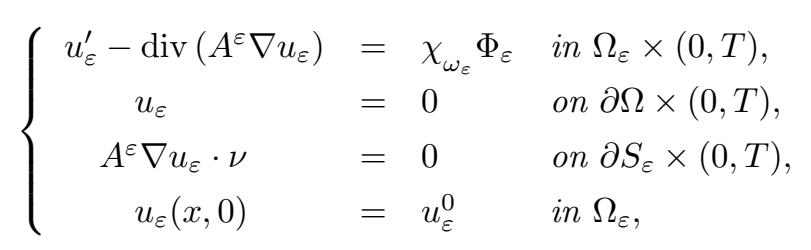

where $\Phi_{\varepsilon}$ is the control given by Theorem 3.3 .

We have, as $\varepsilon \rightarrow 0$, the following convergences:

$$
\begin{aligned}
& \text { i) } \quad \chi_{\omega_{\varepsilon}} \widetilde{\Phi_{\varepsilon}} \rightarrow \chi_{\omega} \theta \Phi \quad \text { weakly in } L^{2}(\Omega \times(0, T)) \text {, } \\
& \text { ii) } P^{\varepsilon} u_{\varepsilon} \rightarrow u \quad \text { weakly in } L^{2}\left(0, T ; H_{0}^{1}(\Omega)\right) \text {, }
\end{aligned}
$$

where $\Phi$ is the solution of (3.10) for $\phi^{0}=\Phi^{0}$, $\Phi^{0}$ being the unique minimum point of the functional $J$ defined by (3.9) and $u$ is the solution of

$$
\left\{\begin{aligned}
\theta u^{\prime}-\operatorname{div}\left(A^{0} \nabla u\right) & =\chi_{\omega} \theta \Phi & & \text { in } \Omega \times(0, T), \\
u & =0 & & \text { on } \partial \Omega \times(0, T), \\
u(x, 0) & =u^{0} & & \text { in } \Omega .
\end{aligned}\right.
$$

Moreover, we have the following approximate controllability:

$$
\left\|u(x, T)-w^{1}\right\|_{L^{2}(\Omega)} \leq \frac{\alpha}{\sqrt{\theta}} .
$$

This theorem will be proved in Section 7. Its proof needs to know the asymptotic behaviour of the controls. This will be studied in Section 6 .

Remark 3.5. Notice that in (3.15) the approximate controllability is altered by a constant which depends on the proportion of material in the reference cell. This is related to the fact that the coerciveness constant of the limit functional of $J_{\varepsilon}$ is multiplied (with respect to that of $J_{\varepsilon}$ ) by $\sqrt{\theta}$. The case where $\theta=1$ corresponds to the case without holes studied by Zuazua (see [14]).

Remark 3.6. Introduce the functional

$$
\widetilde{J}_{\varepsilon}: L^{2}(\Omega) \longrightarrow \mathbb{R}
$$

by

$$
\widetilde{J}_{\varepsilon}\left(\psi^{0}\right)=J_{\varepsilon}\left(\psi_{\mid \Omega_{\varepsilon}}^{0}\right)
$$


where $\psi^{0}{ }_{\mid \Omega_{\varepsilon}}$ is the restriction of $\psi^{0}$ to $\Omega_{\varepsilon}$ and $J_{\varepsilon}$ is the functional given by (3.5). One can show, using Propositions 6.4 and 6.5 of Section 6 , that $\widetilde{J}_{\varepsilon} \Gamma$-converges, in the sense of De Giorgi (see [6]), for the strong $L^{2}(\Omega)$ topology.

On the other hand, the functionals $\widetilde{J}_{\varepsilon}$ are not uniformly coercive (see De Giorgi and Franzoni [7] for the definition and related properties), since a set of the form $\left\{v \in L^{2}(\Omega), \widetilde{J}_{\varepsilon} \leq c\right\}$ is not compact in $L^{2}(\Omega)$. Nevertheless, in Theorem 6.1 we give a direct proof of the convergence of the minimum points.

The last result of this section shows that the solution $u_{\varepsilon}$ of the control problem (3.6) behaves, at the limit, as the solution of the system obtained replacing the right-hand side in (3.6) by the control of the homogenized problem (3.14). Actually, one has

Corollary 3.7. Under the assumptions of Theorem 3.4, let $u_{\varepsilon}$ be the solution of the controllability problem (3.6), $\Phi$ being the control given by Theorem 3.3.

Then, if $z_{\varepsilon}$ is the solution of

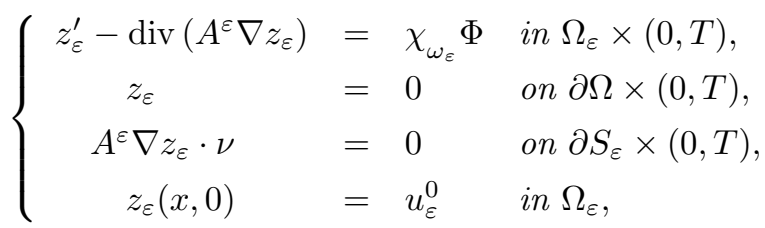

one has, as $\varepsilon \rightarrow 0$, the following convergences:

(i) $\quad P^{\varepsilon} u_{\varepsilon}-P^{\varepsilon} z_{\varepsilon} \rightarrow 0 \quad$ weakly in $L^{2}\left(0, T ; H_{0}^{1}(\Omega)\right)$,

(ii) $\quad \lim _{\varepsilon \rightarrow 0}\left\|u_{\varepsilon}-z_{\varepsilon}\right\|_{C\left([0, T] ; L^{2}\left(\Omega_{\varepsilon}\right)\right)}=0$,

$$
\lim _{\varepsilon \rightarrow 0}\left\|\nabla u_{\varepsilon}-\nabla z_{\varepsilon}\right\|_{L^{2}\left(0, T ;\left[L^{1}\left(\Omega_{\varepsilon}\right)\right]^{n}\right)}=0 .
$$

Moreover

$$
\limsup _{\varepsilon \rightarrow 0}\left\|z_{\varepsilon}(T)-w_{\varepsilon}^{1}\right\|_{L^{2}\left(\Omega_{\varepsilon}\right)} \leq \alpha
$$

This result will be proved in Section 7 .

\section{The CONTROL FOR FIXED $\varepsilon$}

In this section we give, by adapting to our case some technics of Fabre et al. [10,11] and Zuazua [14], some properties of the functional $J_{\varepsilon}$ defined by (3.5). This allows us to characterize the control of system (3.1).

We have the following lemma:

Lemma 4.1. Under assumption (2.3) the functional $J_{\varepsilon}$ defined by (3.5) is continuous, strictly convex and satisfies

$$
\liminf _{\left\|\phi_{\varepsilon}^{0}\right\|_{L^{2}\left(\Omega_{\varepsilon}\right)} \rightarrow \infty} \frac{J_{\varepsilon}\left(\phi_{\varepsilon}^{0}\right)}{\left\|\phi_{\varepsilon}^{0}\right\|_{L^{2}\left(\Omega_{\varepsilon}\right)}} \geq \alpha, \quad \text { for any } \varepsilon>0 \text { fixed } \text {. }
$$

Moreover, if $\Phi_{\varepsilon}^{0}$ is the (unique) minimum point of $J_{\varepsilon}$, we have

$$
\left\|\left(w_{\varepsilon}^{1}-v_{\varepsilon}(T)\right)\right\|_{L^{2}\left(\Omega_{\varepsilon}\right)} \leq \alpha \Longleftrightarrow \Phi_{\varepsilon}^{0}=0 .
$$

We skip here the proof which follows exactly that given in [11], for the study of the approximate controllability of the semilinear heat equation. In Section 3 (Lem. 5.2) we will prove that this inequality holds uniformly in $\varepsilon$. 
We have the following characterization of the minimum point of $J_{\varepsilon}$ :

Corollary 4.2. Under the assumption of Lemma 4.1, for $\varepsilon$ fixed, let $\Phi_{\varepsilon}^{0}$ be the minimum point of the functional $J_{\varepsilon}$. We have

$$
\left|\int_{0}^{T} \int_{\omega_{\varepsilon}} \Phi_{\varepsilon} \Psi_{\varepsilon} d x d t-\int_{\Omega_{\varepsilon}}\left(w_{\varepsilon}^{1}-v_{\varepsilon}(T)\right) \psi_{\varepsilon}^{0} d x\right| \leq \alpha\left\|\psi_{\varepsilon}^{0}\right\|_{L^{2}\left(\Omega_{\varepsilon}\right)},
$$

for any $\psi_{\varepsilon}^{0} \in L^{2}\left(\Omega_{\varepsilon}\right)$, with $\Phi_{\varepsilon}$ solution of (3.3) for $\phi_{\varepsilon}^{0}=\Phi_{\varepsilon}^{0}$ and $\Psi_{\varepsilon}$ solution of (3.3) for $\phi_{\varepsilon}^{0}=\psi_{\varepsilon}^{0}$.

Proof. For $\lambda \in \mathbb{R}_{+}^{*}$ and $\psi_{\varepsilon}^{0} \in L^{2}\left(\Omega_{\varepsilon}\right)$, we have

$$
J_{\varepsilon}\left(\Phi_{\varepsilon}^{0}\right) \leq J_{\varepsilon}\left(\Phi_{\varepsilon}^{0}+\lambda \psi_{\varepsilon}^{0}\right) .
$$

Thus

$$
\begin{aligned}
\int_{\Omega_{\varepsilon}}\left(w_{\varepsilon}^{1}-v_{\varepsilon}(T)\right) \psi_{\varepsilon}^{0} d x & \leq \int_{0}^{T} \int_{\omega_{\varepsilon}} \Phi_{\varepsilon} \Psi_{\varepsilon}+\alpha \liminf _{\lambda \rightarrow 0} \frac{\left\|\Phi_{\varepsilon}^{0}+\lambda \psi_{\varepsilon}^{0}\right\|_{L^{2}\left(\Omega_{\varepsilon}\right)}-\left\|\Phi_{\varepsilon}^{0}\right\|_{L^{2}\left(\Omega_{\varepsilon}\right)}}{\lambda} \\
& \leq \int_{0}^{T} \int_{\omega_{\varepsilon}} \Phi_{\varepsilon} \Psi_{\varepsilon}+\alpha\left\|\psi_{\varepsilon}^{0}\right\|_{L^{2}\left(\Omega_{\varepsilon}\right)} \quad \text { for any } \psi_{\varepsilon}^{0} \in L^{2}\left(\Omega_{\varepsilon}\right) .
\end{aligned}
$$

The same computation with $\lambda \in \mathbb{R}_{-}^{*}$ gives

$$
\int_{\Omega_{\varepsilon}}\left(w_{\varepsilon}^{1}-v_{\varepsilon}(T)\right) \psi_{\varepsilon}^{0} d x \geq \int_{0}^{T} \int_{\omega_{\varepsilon}} \Phi_{\varepsilon} \Psi_{\varepsilon}-\alpha\left\|\psi_{\varepsilon}^{0}\right\|_{L^{2}\left(\Omega_{\varepsilon}\right)} \quad \text { for any } \psi_{\varepsilon}^{0} \in L^{2}\left(\Omega_{\varepsilon}\right) .
$$

Inequalities (4.4) and (4.5) give the result.

Proof of Theorem 3.3. We decompose the solution $u_{\varepsilon}$ as $u_{\varepsilon}=w_{\varepsilon}+v_{\varepsilon}$ where $v_{\varepsilon}$ is defined by (3.4) and $w_{\varepsilon}$ is the solution of the following problem:

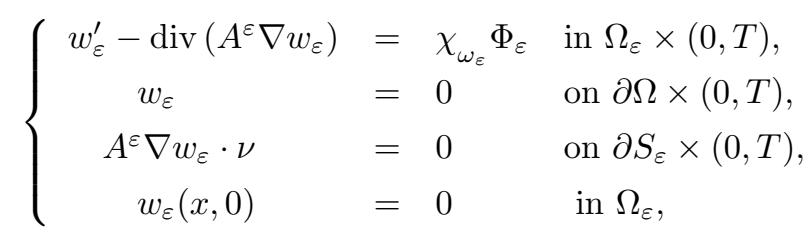

where $\Phi_{\varepsilon}$ is the solution of (3.3) for $\phi_{\varepsilon}^{0}=\Phi_{\varepsilon}^{0}$, $\Phi_{\varepsilon}^{0}$ being the unique minimum point of the functional $J_{\varepsilon}$ defined by (3.5). Let now $\psi_{\varepsilon}^{0} \in L^{2}\left(\Omega_{\varepsilon}\right)$ and let $\Psi_{\varepsilon}$ be the solution of the transposed problem (3.3) for $\phi_{\varepsilon}^{0}=\psi_{\varepsilon}^{0}$. By multiplying (4.6) by $\Psi_{\varepsilon}$ and integrating by parts, we get

$$
-\int_{0}^{T}\left\langle w_{\varepsilon}, \Psi_{\varepsilon}^{\prime}\right\rangle_{V_{\varepsilon}, V_{\varepsilon}^{\prime}} d t+\int_{\Omega_{\varepsilon}} w_{\varepsilon}(T) \Psi_{\varepsilon}(T) d x+\int_{0}^{T} \int_{\Omega_{\varepsilon}} A^{\varepsilon} \nabla w_{\varepsilon} \nabla \Psi_{\varepsilon} d x d t=\int_{0}^{T} \int_{\omega_{\varepsilon}} \Phi_{\varepsilon} \Psi_{\varepsilon} d x d t
$$

From (3.3) and Corollary 4.2, it comes

$$
\left|\int_{\Omega_{\varepsilon}} w_{\varepsilon}(T) \Psi_{\varepsilon}(T) d x-\int_{\Omega_{\varepsilon}}\left(w_{\varepsilon}^{1}-v_{\varepsilon}(T)\right) \psi_{\varepsilon}^{0} d x\right| \leq \alpha\left\|\psi_{\varepsilon}^{0}\right\|_{L^{2}\left(\Omega_{\varepsilon}\right)} .
$$

Since $\Psi_{\varepsilon}(T)=\psi_{\varepsilon}^{0}$ is arbitrary we deduce that

$$
\left\|w_{\varepsilon}(T)-\left(w_{\varepsilon}^{1}-v_{\varepsilon}(T)\right)\right\|_{L^{2}\left(\Omega_{\varepsilon}\right)} \leq \alpha,
$$

which gives

$$
\left\|u_{\varepsilon}(T)-w_{\varepsilon}^{1}\right\|_{L^{2}\left(\Omega_{\varepsilon}\right)} \leq \alpha
$$


Remark 4.3. Let us point out that the symmetry and the $C^{1}$-regularity of the matrix $A$ have been only used when applying the unique continuation property mentioned in Remark 2.2. For the homogenization results recalled in Section 2, symmetry is not necessary and it suffices to suppose $A$ in $\left(L^{\infty}(Y)\right)^{n^{2}}$. Observe also that the unique continuation property is not needed in the case of a control distributed over $\Omega$, i.e. for $\omega=\Omega$.

\section{A priori estimates ON THE CONTROLS}

In this section we give some a priori estimates for the control obtained in Section 4, when the data $w_{\varepsilon}^{1}$ and $u_{\varepsilon}^{0}$ satisfy $(3.11)$, i.e.

$$
\left\{\begin{aligned}
\text { i) } & \lim _{\varepsilon \rightarrow 0}\left\|w_{\varepsilon}^{1}-w^{1}\right\|_{L^{2}\left(\Omega_{\varepsilon}\right)}=0 \\
\text { ii) } & \lim _{\varepsilon \rightarrow 0}\left\|u_{\varepsilon}^{0}-u^{0}\right\|_{L^{2}\left(\Omega_{\varepsilon}\right)}=0
\end{aligned}\right.
$$

Remark 5.1. Under assumption (5.1), using the corrector result stated in Theorem 2.1, we deduce that

$$
\lim _{\varepsilon \rightarrow 0}\left\|\left(w_{\varepsilon}^{1}-v_{\varepsilon}(T)\right)-\left(w^{1}-v(T)\right)\right\|_{L^{2}\left(\Omega_{\varepsilon}\right)}=0
$$

where $v$ is solution of (3.8).

In the same spirit as in [14], we show in the next lemma that inequality (4.1) is uniform in $\varepsilon$. This will provide a uniform estimate in $\varepsilon$ for the unique minimum point $\Phi_{\varepsilon}^{0}$ of the functional $J_{\varepsilon}$.

Lemma 5.2. Under assumptions (2.3) and (5.1), the functional $J_{\varepsilon}$ defined by (3.5) satisfies:

$$
\liminf _{\left\|\phi_{\varepsilon}^{0}\right\|_{L^{2}\left(\Omega_{\varepsilon}\right)} \rightarrow \infty} \frac{J_{\varepsilon}\left(\phi_{\varepsilon}^{0}\right)}{\left\|\phi_{\varepsilon}^{0}\right\|_{L^{2}\left(\Omega_{\varepsilon}\right)}} \geq \alpha
$$

Proof. Consider a sequence $\{j\} \subset \mathbb{N}$ and $\left\{\varepsilon_{j}\right\}_{j \in \mathbb{N}} \subset\{\varepsilon\}_{\varepsilon>0}$ such that

$$
\varepsilon_{j} \rightarrow 0 \quad \text { and } \quad\left\|\phi_{\varepsilon_{j}}^{0}\right\|_{L^{2}\left(\Omega_{\varepsilon_{j}}\right)} \rightarrow \infty \quad \text { as } j \rightarrow \infty
$$

To simplify, we still denote by $\varepsilon$ such a sequence. We have

$$
\left\|\phi_{\varepsilon}^{0}\right\|_{L^{2}\left(\Omega_{\varepsilon}\right)} \rightarrow \infty \quad \text { as } \varepsilon \rightarrow 0
$$

Set $\psi_{\varepsilon}^{0}=\frac{\phi_{\varepsilon}^{0}}{\left\|\phi_{\varepsilon}^{0}\right\|_{L^{2}\left(\Omega_{\varepsilon}\right)}}$. Then, there exists a subsequence (still denoted by $\varepsilon$ ) and $\psi^{0} \in L^{2}(\Omega)$ such that

$$
\widetilde{\psi_{\varepsilon}^{0}} \rightarrow \psi^{0} \quad \text { weakly in } L^{2}(\Omega)
$$

Set $\Psi_{\varepsilon}=\frac{\phi_{\varepsilon}}{\left\|\phi_{\varepsilon}^{0}\right\|_{L^{2}\left(\Omega_{\varepsilon}\right)}}$, where $\phi_{\varepsilon}$ is solution of (3.3). We have

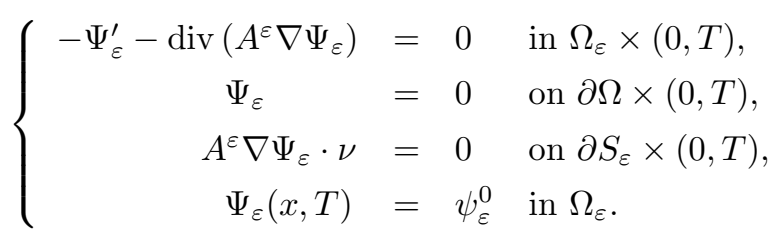

From Theorem 2.1, we have

$$
\widetilde{\Psi_{\varepsilon}} \rightarrow \theta \Psi \quad \text { weakly in } L^{\infty}\left(0, T ; L^{2}(\Omega)\right)
$$


where $\Psi$ is solution of

$$
\left\{\begin{array}{clll}
-\theta \Psi^{\prime}-\operatorname{div}\left(A^{0} \nabla \Psi\right) & =0 & & \text { in } \Omega \times(0, T), \\
\Psi & = & 0 & \text { on } \partial \Omega \times(0, T), \\
\Psi(x, T) & =\frac{\psi^{0}}{\theta} & \text { in } \Omega .
\end{array}\right.
$$

From the definitions of $J_{\varepsilon}$ (see $\left.(3.5)\right), \psi_{\varepsilon}^{0}$ and $\Psi_{\varepsilon}$, we have

$$
\frac{J_{\varepsilon}\left(\phi_{\varepsilon}^{0}\right)}{\left\|\phi_{\varepsilon}^{0}\right\|_{L^{2}\left(\Omega_{\varepsilon}\right)}}=\frac{1}{2}\left\|\phi_{\varepsilon}^{0}\right\|_{L^{2}\left(\Omega_{\varepsilon}\right)} \int_{0}^{T} \int_{\omega}\left|\widetilde{\Psi_{\varepsilon}}\right|^{2} d x d t+\alpha-\int_{\Omega}\left(\widetilde{w_{\varepsilon}^{1}}-\widetilde{v_{\varepsilon}(T)}\right) \widetilde{\psi_{\varepsilon}^{0}} d x .
$$

Two cases arise

$$
\begin{aligned}
& \text { (i) } \liminf _{\varepsilon \rightarrow 0} \int_{0}^{T} \int_{\omega}\left|\widetilde{\Psi_{\varepsilon}}\right|^{2} d x d t>0, \\
& \text { (ii) } \liminf _{\varepsilon \rightarrow 0} \int_{0}^{T} \int_{\omega}\left|\widetilde{\Psi_{\varepsilon}}\right|^{2} d x d t=0 .
\end{aligned}
$$

In case (i), in view of (5.1) and (5.4), the term $\int_{\Omega}\left(\widetilde{w_{\varepsilon}^{1}}-\widetilde{v_{\varepsilon}(T)}\right) \widetilde{\psi_{\varepsilon}^{0}} d x$ is uniformly bounded with respect to $\varepsilon$. Hence, one has

$$
\lim _{\varepsilon \rightarrow 0} \frac{J_{\varepsilon}\left(\phi_{\varepsilon}^{0}\right)}{\left\|\phi_{\varepsilon}^{0}\right\|_{L^{2}\left(\Omega_{\varepsilon}\right)}}=+\infty
$$

which gives (5.3).

For case (ii), since $\theta \neq 0$, we deduce from (5.6) that

$$
\Psi=0 \quad \text { in } \omega \times(0, T) .
$$

From the unique-continuation property due to Saut and Scheurer [13], we deduce that

$$
\Psi=0 \quad \text { in } \Omega \times(0, T)
$$

Since $\Psi \in C\left([0, T] ; L^{2}(\Omega)\right)$ and due to $(5.7)$, we have $\Psi(T)=\frac{\psi^{0}}{\theta}$. Then we deduce that

$$
\psi^{0}=0 \quad \text { in } L^{2}(\Omega)
$$

On the other hand, from $(5.2,5.4,5.9)$ and Remark 2.2 (written for $\varphi_{\varepsilon}=\psi_{\varepsilon}^{0}, u_{\varepsilon}^{0}=w_{\varepsilon}^{1}-v_{\varepsilon}(T)$ and $\left.u^{0}=\theta\left(w^{1}-v(T)\right)\right)$, it comes that

$$
\lim _{\varepsilon \rightarrow 0} \int_{\Omega}\left(\widetilde{w_{\varepsilon}^{1}}-\widetilde{v_{\varepsilon}(T)}\right) \widetilde{\psi_{\varepsilon}^{0}} d x=0
$$

Passing to the limit as $\varepsilon \rightarrow 0$ in (5.8), we find

$$
\liminf _{\varepsilon \rightarrow 0} \frac{J_{\varepsilon}\left(\phi_{\varepsilon}^{0}\right)}{\left\|\phi_{\varepsilon}^{0}\right\|_{L^{2}\left(\Omega_{\varepsilon}\right)}} \geq \alpha
$$

which shows that we have again (5.3) and ends the proof.

Corollary 5.3. Under the assumptions of Lemma 5.2, there exists a constant c independent of $\varepsilon$ such that

$$
\left\|\Phi_{\varepsilon}^{0}\right\|_{L^{2}\left(\Omega_{\varepsilon}\right)} \leq c
$$


Proof. By contradiction, we suppose that there exists a subsequence (still denoted by $\varepsilon$ ), such that

$$
\left\|\Phi_{\varepsilon}^{0}\right\|_{L^{2}\left(\Omega_{\varepsilon}\right)} \longrightarrow \infty, \quad \text { as } \varepsilon \rightarrow 0
$$

so that, from Lemma 5.2,

$$
\lim _{\varepsilon \rightarrow 0} \frac{J_{\varepsilon}\left(\Phi_{\varepsilon}^{0}\right)}{\left\|\Phi_{\varepsilon}^{0}\right\|_{L^{2}\left(\Omega_{\varepsilon}\right)}} \geq \alpha
$$

Since $\Phi_{\varepsilon}^{0}$ minimizes the functional $J_{\varepsilon}$ one has

$$
J_{\varepsilon}\left(\Phi_{\varepsilon}^{0}\right) \leq J_{\varepsilon}(0)=0 \quad \forall \varepsilon
$$

Hence,

$$
\alpha \leq \lim _{\varepsilon \rightarrow 0} \frac{J_{\varepsilon}\left(\Phi_{\varepsilon}^{0}\right)}{\left\|\Phi_{\varepsilon}^{0}\right\|_{L^{2}\left(\Omega_{\varepsilon}\right)}} \leq 0
$$

which contradicts the fact that $\alpha$ is strictly positive.

Remark 5.4. From estimate (5.11) we deduce that there exists $\Phi^{0}$ and a subsequence (still denoted by $\varepsilon$ ) such that

$$
\widetilde{\Phi_{\varepsilon}^{0}} \rightarrow \theta \Phi^{0} \quad \text { weakly in } L^{2}(\Omega)
$$

One can then apply Theorem 2.1 to the controls $\left\{\Phi_{\varepsilon}\right\}_{\varepsilon}$, solutions of (3.3) for $\phi_{\varepsilon}^{0}=\Phi_{\varepsilon}^{0}$. All the difficulty is to identify the weak limit $\Phi^{0}$ as the unique minimum point of a suitable functional. In this case, convergence (5.12) holds for the whole sequence $\left\{\Phi_{\varepsilon}^{0}\right\}_{\varepsilon>0}$.

\section{SOME PRELIMINARY RESUlts}

In this section we study the limit behaviour of the control $\Phi_{\varepsilon}$ given by Theorem 3.3. The following theorem shows that the limit control can be uniquely identified as the solution of the transposed problem (3.10) associated to the minimum point of the functional (3.9).

Theorem 6.1. Under hypothesis (2.3) and (5.1), let $u^{0}$ and $w^{1}$ be given in $L^{2}(\Omega)$. The sequence $\left\{\Phi_{\varepsilon}^{0}\right\}_{\varepsilon}$ of the minimum points associated to the functionals $J_{\varepsilon}$, satisfies, as $\varepsilon \rightarrow 0$, the following convergences:

$$
\left\{\begin{array}{l}
\text { i) } \quad \widetilde{\Phi_{\varepsilon}^{0}} \rightarrow \theta \Phi^{0} \quad \text { weakly in } L^{2}(\Omega) \\
\text { ii) } \quad\left\|\Phi_{\varepsilon}^{0}\right\|_{L^{2}\left(\Omega_{\varepsilon}\right)} \longrightarrow \sqrt{\theta}\left\|\Phi^{0}\right\|_{L^{2}(\Omega)}
\end{array}\right.
$$

where $\Phi^{0}$ is the minimum point of the functional $J$ defined by (3.9).

The proof of this theorem will be given at the end of this section. It makes use of Propositions 6.4 and 6.5 below.

An immediate consequence of Theorem 6.1 is the following:

Corollary 6.2. Under the hypothesis of Theorem 6.1 the control $\Phi_{\varepsilon}$ given by Theorem 3.3 satisfies, as $\varepsilon \rightarrow 0$,

$$
\left\{\begin{array}{ccc}
\text { (i) } & P^{\varepsilon} \Phi_{\varepsilon} \rightarrow \Phi & \text { weakly in } L^{2}\left(0, T ; H_{0}^{1}(\Omega)\right) \\
\text { (ii) } & \widetilde{\Phi_{\varepsilon}} \rightarrow \theta \Phi & \text { weakly in } L^{\infty}\left(0, T ; L^{2}(\Omega)\right)
\end{array}\right.
$$

with $\Phi$ solution of (3.10) for $\phi^{0}=\Phi^{0}$, $\Phi^{0}$ being the minimum point of the functional $J$ given by (3.9). 
Moreover, we have the following corrector result:

$$
\left\{\begin{array}{ccc}
\text { (i) } & \lim _{\varepsilon \rightarrow 0}\left\|\Phi_{\varepsilon}-\Phi\right\|_{C\left([0, T] ; L^{2}\left(\Omega_{\varepsilon}\right)\right)} & =0, \\
\text { (ii) } \lim _{\varepsilon \rightarrow 0}\left\|\nabla \Phi_{\varepsilon}-C^{\varepsilon} \nabla \Phi\right\|_{L^{2}\left(0, T ;\left[L^{1}\left(\Omega_{\varepsilon}\right)\right]^{n}\right)} & =0,
\end{array}\right.
$$

where $C^{\varepsilon}(x)$ is the corrector matrix given by (2.10).

Proof. Thanks to convergences (6.1) given by Theorem 6.1, the result is a direct consequence of the homogenization result given in Theorem 2.1, applied to the control $\Phi_{\varepsilon}$.

Remark 6.3. As for the functional $J_{\varepsilon}$ given by (3.5), one can easily show that the functional $J\left(\phi^{0}\right)$ defined by (3.9) is continuous, strictly convex and satisfies the following coercivity condition:

$$
\liminf _{\left\|\phi^{0}\right\|_{L^{2}(\Omega)} \rightarrow \infty} \frac{J\left(\phi^{0}\right)}{\left\|\phi^{0}\right\|_{L^{2}(\Omega)}} \geq \alpha \sqrt{\theta}
$$

Moreover, if $\Phi^{0} \in L^{2}(\Omega)$ is such that

$$
J\left(\Phi^{0}\right)=\min _{\phi^{0} \in L^{2}(\Omega)} J\left(\phi^{0}\right),
$$

we have

$$
\left|\int_{0}^{T} \int_{\Omega} \theta \Psi \Phi-\int_{\Omega} \theta\left(w^{1}-v(T)\right) \psi^{0}\right| \leq \alpha \sqrt{\theta}\left\|\psi^{0}\right\|_{L^{2}(\Omega)},
$$

for any $\psi^{0} \in L^{2}(\Omega)$, with $\Phi$ and $\Psi$ solutions of (3.10) for $\phi^{0}=\Phi^{0}$ and $\phi^{0}=\psi^{0}$ respectively.

Proposition 6.4. Let $\psi^{0} \in L^{2}(\Omega)$. Under hypothesis (2.3) and (5.1) the functional $J_{\varepsilon}$ defined by (3.5) satisfies

$$
\lim _{\varepsilon \rightarrow 0} J_{\varepsilon}\left(\psi^{0} \Omega_{\varepsilon}\right)=J\left(\psi^{0}\right)
$$

where $\psi^{0}{ }_{\mid \Omega_{\varepsilon}}$ is the restriction of $\psi^{0}$ to $\Omega_{\varepsilon}$ and $J$ is the functional given by (3.9).

Proof. Let $\psi^{0} \in L^{2}(\Omega)$ and $\Psi_{\varepsilon}(x, t)$ the solution of the following problem

$$
\left\{\begin{aligned}
-\Psi_{\varepsilon}^{\prime}-\operatorname{div}\left(A^{\varepsilon} \nabla \Psi_{\varepsilon}\right) & =0 & & \text { in } \Omega_{\varepsilon} \times(0, T), \\
\Psi_{\varepsilon} & =0 & & \text { on } \partial \Omega \times(0, T), \\
A^{\varepsilon} \nabla \Psi_{\varepsilon} \cdot \nu & =0 & & \text { on } \partial S_{\varepsilon} \times(0, T), \\
\Psi_{\varepsilon}(x, T) & =\psi^{0}{ }_{\mid \Omega_{\varepsilon}} & & \text { in } \Omega_{\varepsilon} .
\end{aligned}\right.
$$

Since

$$
\widetilde{\psi^{0} \Omega_{\varepsilon}} \rightarrow \theta \psi^{0} \quad \text { weakly in } L^{2}(\Omega),
$$

from Theorem 2.1 applied to $\Psi_{\varepsilon}$, we have

$$
P^{\varepsilon} \Psi_{\varepsilon} \rightarrow \Psi \quad \text { weakly in } L^{2}\left(0, T ; H_{0}^{1}(\Omega)\right),
$$

where $\Psi$ is solution of

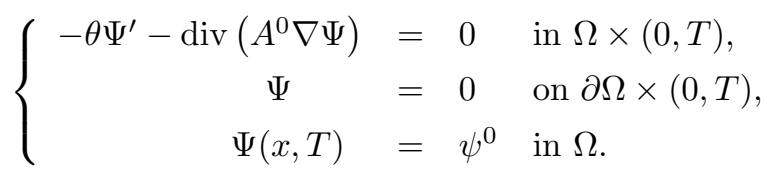


We want to pass to the limit in the following expression:

$$
J_{\varepsilon}\left(\psi_{\mid \Omega_{\varepsilon}}^{0}\right)=\frac{1}{2} \int_{0}^{T} \int_{\omega}\left|\widetilde{\Psi_{\varepsilon}}\right|^{2} d x d t+\alpha\left(\int_{\Omega} \chi_{\Omega_{\varepsilon}}\left|\psi^{0}\right|^{2}\right)^{\frac{1}{2}} d x-\int_{\Omega}\left(\widetilde{w_{\varepsilon}^{1}}-\widetilde{v_{\varepsilon}(T)}\right) \psi^{0} d x
$$

It is easy to see, in virtue of (2.2), that

$$
\lim _{\varepsilon \rightarrow 0}\left(\int_{\Omega} \chi_{\Omega_{\varepsilon}}\left|\psi^{0}\right|^{2} d x\right)^{\frac{1}{2}} \longrightarrow\left(\int_{\Omega} \theta\left|\psi^{0}\right|^{2} d x\right)^{\frac{1}{2}}
$$

Taking into account (5.2) and Remark 2.2, we get

$$
\int_{\Omega}\left(\widetilde{w_{\varepsilon}^{1}}-\widetilde{v_{\varepsilon}(T)}\right) \psi^{0} d x \longrightarrow \int_{\Omega} \theta\left(w^{1}-v(T)\right) \psi^{0} d x
$$

We show now that

$$
\lim _{\varepsilon \rightarrow 0} \int_{0}^{T} \int_{\omega}\left|\widetilde{\Psi_{\varepsilon}}\right|^{2} d x d t=\int_{0}^{T} \int_{\omega} \theta|\Psi|^{2} d x d t
$$

Indeed, we write

$$
\lim _{\varepsilon \rightarrow 0} \int_{0}^{T} \int_{\omega}\left|\widetilde{\Psi_{\varepsilon}}\right|^{2} d x d t=\lim _{\varepsilon \rightarrow 0}\left\langle\widetilde{\Psi_{\varepsilon}}, P^{\varepsilon} \Psi_{\varepsilon}\right\rangle_{L^{2}\left(0, T ; H^{-1}(\Omega)\right), L^{2}\left(0, T ; H_{0}^{1}(\Omega)\right)} .
$$

On the other hand, convergence (2.13) from Theorem 2.1 applied to $\Psi^{\varepsilon}$, gives

$$
\widetilde{\Psi^{\varepsilon}} \longrightarrow \theta \Psi \quad \text { strongly in } \mathcal{C}\left([0, T] ; H^{-1}(\Omega)\right) \text {. }
$$

This convergence and (6.8) allows us to pass to the limit and we have

$$
\lim _{\varepsilon \rightarrow 0} \int_{0}^{T} \int_{\omega}\left|\widetilde{\Psi_{\varepsilon}}\right|^{2} d x d t=\int_{0}^{T} \int_{\omega} \theta|\Psi|^{2} d x d t
$$

Finally, convergences $(6.11,6.12)$ and $(6.13)$ give

$$
\begin{aligned}
\lim _{\varepsilon \rightarrow 0} J_{\varepsilon}\left(\psi^{0}{ }_{\mid \Omega_{\varepsilon}}\right) & =\frac{1}{2} \int_{0}^{T} \int_{\omega} \theta|\Psi(x, t)|^{2} d x d t+\alpha\left(\int_{\Omega} \theta\left|\psi^{0}\right|^{2}\right)^{\frac{1}{2}} d x-\int_{\Omega} \theta\left(w^{1}-v(T)\right) \psi^{0} d x \\
& =J\left(\psi^{0}\right),
\end{aligned}
$$

where $J$ is the functional defined by (3.9).

Proposition 6.5. Let $\psi^{0} \in L^{2}(\Omega)$. Under hypothesis of Proposition 6.4, for any sequence $\left\{\psi_{\varepsilon}^{0}\right\}_{\varepsilon}$ $\subset L^{2}\left(\Omega_{\varepsilon}\right)$ such that, as $\varepsilon \rightarrow 0$,

$$
\widetilde{\psi_{\varepsilon}^{0}} \rightarrow \theta \psi^{0} \quad \text { weakly in } L^{2}(\Omega)
$$

we have

$$
\liminf _{\varepsilon \rightarrow 0} J_{\varepsilon}\left(\psi_{\varepsilon}^{0}\right) \geq J\left(\psi^{0}\right)
$$

To prove this proposition, we make use of the following lemma, which we give in a more general framework than needed here. 
Lemma 6.6. Let $\mathcal{O}$ be an open set of $\mathbb{R}^{n}$ and $\left\{\mathcal{O}_{\varepsilon}\right\}_{\varepsilon} \subset \mathcal{O}$ a sequence of open subsets of $\mathcal{O}$. Suppose that $\left\{v_{\varepsilon}\right\}_{\varepsilon} \subset L^{p}\left(\mathcal{O}_{\varepsilon}\right), p>1$, is such that, as $\varepsilon \rightarrow 0$,

1. $\chi_{\mathcal{O}_{\varepsilon}} \rightarrow \chi_{0}$ in $L^{\infty}(\mathcal{O})$ weakly $*$,

2. $\widetilde{v_{\varepsilon}} \rightarrow \chi_{0} v$ weakly in $L^{p}(\mathcal{O})$,

for some $v \in L^{p}(\mathcal{O})$. Then

$$
\liminf _{\varepsilon \rightarrow 0} \int_{\mathcal{O}_{\varepsilon}}\left|v_{\varepsilon}\right|^{p} d x \geq \int_{\mathcal{O}} \chi_{0}|v|^{p} d x
$$

Proof. By the convexity on $\mathbb{R}$ of the function $g(y)=|y|^{p}$, we have

$$
\left|v_{\varepsilon}(x)\right|^{p} \geq|v(x)|^{p}+p|v(x)|^{p-2} v(x)\left(v_{\varepsilon}(x)-v(x)\right) \quad \text { a.e. on } \mathcal{O}_{\varepsilon} .
$$

Hence, passing to the zero extensions, we get

$$
\int_{\mathcal{O}}\left|\widetilde{v_{\varepsilon}}\right|^{p} d x \geq \int_{\mathcal{O}} \chi_{\mathcal{O}_{\varepsilon}}|v|^{p} d x+p \int_{\mathcal{O}}|v|^{p-2} v\left(\widetilde{v_{\varepsilon}}-v \chi_{\mathcal{O}_{\varepsilon}}\right) d x .
$$

On the other hand, it is easy to see that the assumptions imply that

$$
\begin{array}{r}
\widetilde{v_{\varepsilon}}-v \chi_{\mathcal{O}_{\varepsilon}} \rightarrow 0 \quad \text { weakly in } L^{p}(\mathcal{O}), \\
|v|^{p-2} v \in L^{p^{\prime}}(\mathcal{O}), \text { where } \quad \frac{1}{p}+\frac{1}{p^{\prime}}=1,
\end{array}
$$

so we deduce the following convergences:

$$
\begin{aligned}
\int_{\mathcal{O}}|v|^{p-2} v\left(\widetilde{v}_{\varepsilon}-v \chi_{\mathcal{O}_{\varepsilon}}\right) d x & \longrightarrow 0 \\
\int_{\mathcal{O}} \chi_{\mathcal{O}_{\varepsilon}}|v|^{p} d x & \longrightarrow \int_{\mathcal{O}} \chi_{0}|v|^{p} d x .
\end{aligned}
$$

Then, by passing to the lower limit, as $\varepsilon \rightarrow 0$, in (6.17) one has the result.

Remark 6.7. Observe that inequality (6.16) improves that obtained by using the lower semicontinuity of the norm, namely

since $\chi_{0} \leq 1$.

$$
\liminf \int_{\mathcal{O}}\left|\widetilde{v}_{\varepsilon}\right|^{p} d x \geq \int_{\mathcal{O}} \chi_{0}^{p}|v|^{p} d x
$$

Inequality (6.16) will play an essential role on order to identify the limit of the sequence $\left(\Phi_{\varepsilon}^{0}\right)$ of the minimum points of the functionals $J_{\varepsilon}$.

Proof of Proposition 6.5. By applying Lemma 6.6 for $\mathcal{O}=\Omega, \mathcal{O}_{\varepsilon}=\Omega_{\varepsilon}$ and $v_{\varepsilon}=\psi_{\varepsilon}^{0}$, we obtain, by taking into account (2.2), the following inequality:

$$
\liminf _{\varepsilon \rightarrow 0} \int_{\Omega_{\varepsilon}}\left|\psi_{\varepsilon}^{0}\right|^{2} d x \geq \int_{\Omega} \theta\left|\psi^{0}\right|^{2} d x
$$

Let $\Psi_{\varepsilon}$ be the solution of (3.3) for $\phi_{\varepsilon}^{0}=\psi_{\varepsilon}^{0}$. Then from Theorem 2.1 we have

$$
P^{\varepsilon} \Psi_{\varepsilon} \rightarrow \Psi \quad \text { weakly in } L^{2}\left(0, T ; H_{0}^{1}(\Omega)\right),
$$

with $\Psi$ solution of (6.9) and (see (6.13))

$$
\lim _{\varepsilon \rightarrow 0} \int_{0}^{T} \int_{\Omega_{\varepsilon}}\left|\Psi_{\varepsilon}\right|^{2} d x d t=\theta \int_{0}^{T} \int_{\Omega}|\Psi|^{2} d x d t
$$


On the other hand, we deduce from $(5.2,6.14)$ and Remark 2.2 that

$$
\lim _{\varepsilon \rightarrow 0} \int_{\Omega_{\varepsilon}}\left(w_{\varepsilon}^{1}-v_{\varepsilon}(T)\right) \psi_{\varepsilon}^{0} d x=\lim _{\varepsilon \rightarrow 0} \int_{\Omega}\left(\widetilde{w_{\varepsilon}^{1}}-\widetilde{v_{\varepsilon}(T)}\right) \widetilde{\psi_{\varepsilon}^{0}} d x=\int_{\Omega} \theta\left(w^{1}-v(T)\right) \psi^{0} d x
$$

Combining $(6.18,6.19)$ and $(6.20)$ we get

$$
\begin{aligned}
\liminf _{\varepsilon \rightarrow 0} J_{\varepsilon}\left(\psi_{\varepsilon}^{0}\right) & =\liminf _{\varepsilon \rightarrow 0}\left\{\frac{1}{2} \int_{0}^{T} \int_{\omega_{\varepsilon}}\left|\Psi_{\varepsilon}\right|^{2} d x d t+\alpha\left\|\psi_{\varepsilon}^{0}\right\|_{L^{2}\left(\Omega_{\varepsilon}\right)}-\int_{\Omega_{\varepsilon}}\left(w_{\varepsilon}^{1}-v_{\varepsilon}(T)\right) \psi_{\varepsilon}^{0} d x,\right\} \\
& \geq \frac{1}{2} \theta \int_{0}^{T} \int_{\omega}|\Psi|^{2} d x d t+\alpha \sqrt{\theta}\left\|\psi^{0}\right\|_{L^{2}(\Omega)}-\int_{\Omega} \theta\left(w^{1}-v(T)\right) \psi^{0} d x,
\end{aligned}
$$

which is the desired result.

Proof of Theorem 6.1. As seen in Remark 5.4, the sequence $\left\{\Phi_{\varepsilon}^{0}\right\}_{\varepsilon}$ of the minimum points of $J_{\varepsilon}$, satisfies (up to a subsequence),

$$
\widetilde{\Phi_{\varepsilon}^{0}} \rightarrow \theta \Phi^{0} \quad \text { weakly in } L^{2}(\Omega)
$$

From Proposition 6.5, we deduce

$$
J\left(\Phi^{0}\right) \leq \liminf _{\varepsilon \rightarrow 0} J_{\varepsilon}\left(\Phi_{\varepsilon}^{0}\right)
$$

On the other hand, let $\psi^{0}$ be in $L^{2}(\Omega)$. Using Proposition 6.4 , we have

$$
\limsup _{\varepsilon \rightarrow 0} J_{\varepsilon}\left(\Phi_{\varepsilon}^{0}\right) \leq \limsup _{\varepsilon \rightarrow 0} J_{\varepsilon}\left(\psi^{0}{ }_{\mid \Omega_{\varepsilon}}\right)=\lim _{\varepsilon \rightarrow 0} J_{\varepsilon}\left(\psi^{0}{ }_{\mid \Omega_{\varepsilon}}\right)=J\left(\psi^{0}\right) .
$$

From (6.23) and (6.24) and Remark 6.3, we deduce that $\Phi^{0}$ is the unique minimum point of the functional $J$ given by (3.9). Consequently all the sequence $\left\{\widetilde{\Phi_{\varepsilon}^{0}}\right\}_{\varepsilon}$ weakly converges to $\theta \Phi^{0}$ in $L^{2}(\Omega)$, which gives (6.1)i.

To prove (6.1)ii, we use (6.23) and we choose in $(6.24) \psi^{0}=\Phi^{0}$. We get

$$
J\left(\Phi^{0}\right) \leq \liminf _{\varepsilon \rightarrow 0} J_{\varepsilon}\left(\Phi_{\varepsilon}^{0}\right) \leq \limsup _{\varepsilon \rightarrow 0} J_{\varepsilon}\left(\Phi_{\varepsilon}^{0}\right)=J\left(\Phi^{0}\right),
$$

so

$$
\lim _{\varepsilon \rightarrow 0} J_{\varepsilon}\left(\Phi_{\varepsilon}^{0}\right)=J\left(\Phi^{0}\right)
$$

Moreover, writing (6.13) for the solution $\Phi_{\varepsilon}$ of

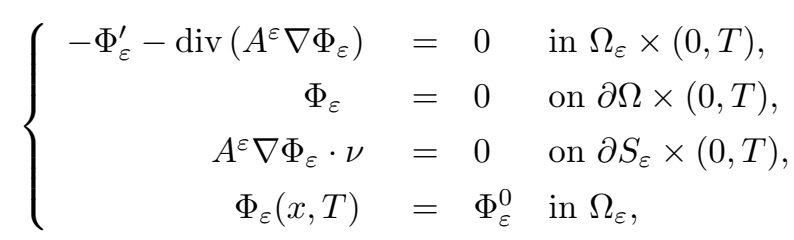

it comes that

$$
\lim _{\varepsilon \rightarrow 0} \int_{0}^{T} \int_{\omega}\left|\widetilde{\Phi_{\varepsilon}}\right|^{2} d x d t=\int_{0}^{T} \int_{\omega} \theta|\Phi|^{2} d x d t
$$

where $\Phi$ is solution of (3.10) for $\phi^{0}=\Phi^{0}$. By virtue of (5.2) and Remark 2.2, we have

$$
\lim _{\varepsilon \rightarrow 0} \int_{\Omega_{\varepsilon}}\left(w_{\varepsilon}^{1}-v_{\varepsilon}(T)\right) \Phi_{\varepsilon}^{0} d x=\lim _{\varepsilon \rightarrow 0} \int_{\Omega}\left(\widetilde{w_{\varepsilon}^{1}}-\widetilde{v_{\varepsilon}(T)}\right) \widetilde{\Phi_{\varepsilon}^{0}} d x=\int_{\Omega} \theta\left(w^{1}-v(T)\right) \Phi^{0} d x
$$


Taking into account $(6.25,6.26)$ and $(6.27)$, we deduce finally

$$
\lim _{\varepsilon \rightarrow 0}\left\|\Phi_{\varepsilon}^{0}\right\|_{L^{2}\left(\Omega_{\varepsilon}\right)}=\sqrt{\theta}\left\|\Phi^{0}\right\|_{L^{2}(\Omega)} .
$$

This ends the proof of Theorem 6.1.

\section{Proofs of Theorem 3.4 And Corollary 3.7}

Proof of Theorem 3.4. Let us notice first that (3.12) is a consequence of Corollary 6.2. On the other hand, as in the proof of Theorem 3.3, we decompose the solution $u_{\varepsilon}$ as $u_{\varepsilon}=v_{\varepsilon}+w_{\varepsilon}, v_{\varepsilon}$ and $w_{\varepsilon}$ being defined respectively by (3.4) and (4.6). Then, the linearity of the extension operator $P^{\varepsilon}$, assumption (3.11) and Theorem 2.1 give

$$
P^{\varepsilon} u_{\varepsilon}=P^{\varepsilon} v_{\varepsilon}+P^{\varepsilon} w_{\varepsilon} \rightarrow u=v+w \quad \text { weakly in } L^{2}\left(0, T ; H_{0}^{1}(\Omega)\right),
$$

where $v$ is the solution of (3.8) and $w$ satisfies the following equation

$$
\left\{\begin{aligned}
\theta w^{\prime}-\operatorname{div}\left(A^{0} \nabla w\right) & =\chi_{\omega} \theta \Phi & & \text { in } \Omega \times(0, T), \\
w & =0 & & \text { on } \partial \Omega \times(0, T), \\
w(x, 0) & =0 & & \text { in } \Omega .
\end{aligned}\right.
$$

This gives convergence (3.13) with $u$ the unique solution of (3.14).

To show (3.15), we consider $\psi^{0} \in L^{2}(\Omega)$ and $\Psi$ solution of (3.10) for $\phi^{0}=\psi^{0}$.

Multiplying equation (7.1) by $\Psi$ and integrating by parts, it comes

$$
\int_{0}^{T} \int_{\Omega}\left(-\theta \Psi^{\prime}-\operatorname{div}\left(A^{0} \nabla \Psi\right)\right) w d x d t+\int_{\Omega} \theta w(T) \Psi(T) d x=\int_{0}^{T} \int_{\omega} \theta \Phi \Psi d x d t
$$

so that

$$
\int_{\Omega} \theta w(T) \Psi(T) d x=\int_{0}^{T} \int_{\omega} \theta \Phi \Psi d x d t
$$

By replacing $\Psi(T)$ by $\psi^{0}$ and using Remark 6.3 we have

$$
\left|\theta \int_{\Omega} w(T) \psi^{0} d x-\theta \int_{\Omega}\left(w^{1}-v(T)\right) \psi^{0} d x\right| \leq \alpha \sqrt{\theta}\left\|\psi^{0}\right\|_{L^{2}(\Omega)} .
$$

Thus,

$$
\left\|w(T)-\left(w^{1}-v(T)\right)\right\|_{L^{2}(\Omega)} \leq \frac{\alpha}{\sqrt{\theta}},
$$

which gives (3.15), since $u(T)=w(T)+v(T)$.

Proof of Corollary 3.7. Observe that the function $\zeta_{\varepsilon}=u_{\varepsilon}-z_{\varepsilon}$ solves the problem

$$
\left\{\begin{array}{clll}
\zeta_{\varepsilon}^{\prime}-\operatorname{div}\left(A^{\varepsilon} \nabla \zeta_{\varepsilon}\right) & = & \rho_{\varepsilon} & \text { in } \Omega_{\varepsilon} \times(0, T), \\
\zeta_{\varepsilon} & = & 0 & \text { on } \partial \Omega \times(0, T), \\
A^{\varepsilon} \nabla \zeta_{\varepsilon} \cdot \nu & = & 0 & \text { on } \partial S_{\varepsilon} \times(0, T), \\
\zeta_{\varepsilon}(x, 0) & = & 0 & \text { in } \Omega_{\varepsilon},
\end{array}\right.
$$

where $\rho_{\varepsilon}=\chi_{\omega_{\varepsilon}}\left(\Phi_{\varepsilon}-\Phi\right)$. On the other hand, Corollary 6.2 gives the convergences

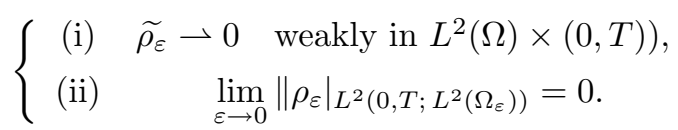


Hence, convergences (3.16) follow from Theorem 2.1. Finally, convergences (3.16)ii together with the estimate (3.7), imply (3.17).

\section{REFERENCES}

[1] C. Brizzi and J.P. Chalot, Homogénéisation dans des ouverts à frontière fortement oscillante. Thèse à l'Université de Nice (1978).

[2] D. Cioranescu and P. Donato, Exact internal controllability in perforated domains. J. Math. Pures Appl. 319 (1989) $185-213$.

[3] D. Cioranescu and P. Donato, An introduction to Homogenization. Oxford University Press (1999).

[4] D. Cioranescu and J. Saint Jean Paulin, Homogenization in open sets with holes. J. Math. Anal. Appl. 319 (1979) 509-607.

[5] R. Dautray and J.-L. Lions, Analyse Mathématique et Calcul Numérique pour les Sciences et Techniques. Masson, Tome 3, Paris (1985).

[6] E. De Giorgi, Sulla convergenza di alcune successioni di integrali del tipo dell'area. Rend. Mat. 4 (1975) $277-294$.

[7] E. De Giorgi and T. Franzoni, Su un tipo di convergenza variazionale. Atti. Accad. Naz. Lincei Cl. Sci. Fis. Mat. Natur. Rend. Lincei (8) $\mathbf{5 8}$ (1975) 842-850.

[8] P. Donato and A. Nabil, Homogénéisation et contrôlabilité approchée de l'équation de la chaleur dans des domaines perforés. C. R. Acad. Sci. Paris Sér. I Math. 324 (1997) 789-794.

[9] P. Donato and A. Nabil, Homogenization and correctors for heat equation in perforated domains. Ricerche di Matematica (to appear).

[10] C. Fabre, J.P. Puel and E. Zuazua, Contrôlabilité approchée de l'équation de la chaleur semilinéaire. C. R. Acad. Sci. Paris Sér. I Math. 314 (1992) 807-812.

[11] C. Fabre, J.P. Puel and E. Zuazua, Approximate controllability for the semilinear heat equation. Proc. Roy. Soc. Edinburgh Sect. A 125 (1995) 31-61.

[12] J.-L. Lions, Remarques sur la contrôlabilité approchée, in Jornadas Hispano-Francesas sobre Control de Sistemas Distribuidos, octubre 1990. Grupo de Análisis Matemático Aplicado de la University of Málaga, Spain (1991) 77-87.

[13] J.-C. Saut and B. Scheurer, Unique continuation for some evolution equations. J. Differential Equations 66 (1987) 118-139.

[14] E. Zuazua, Approximate controllability for linear parabolic equations with rapidly oscillating coefficients. Control Cybernet. 23 (1994) 1-8. 